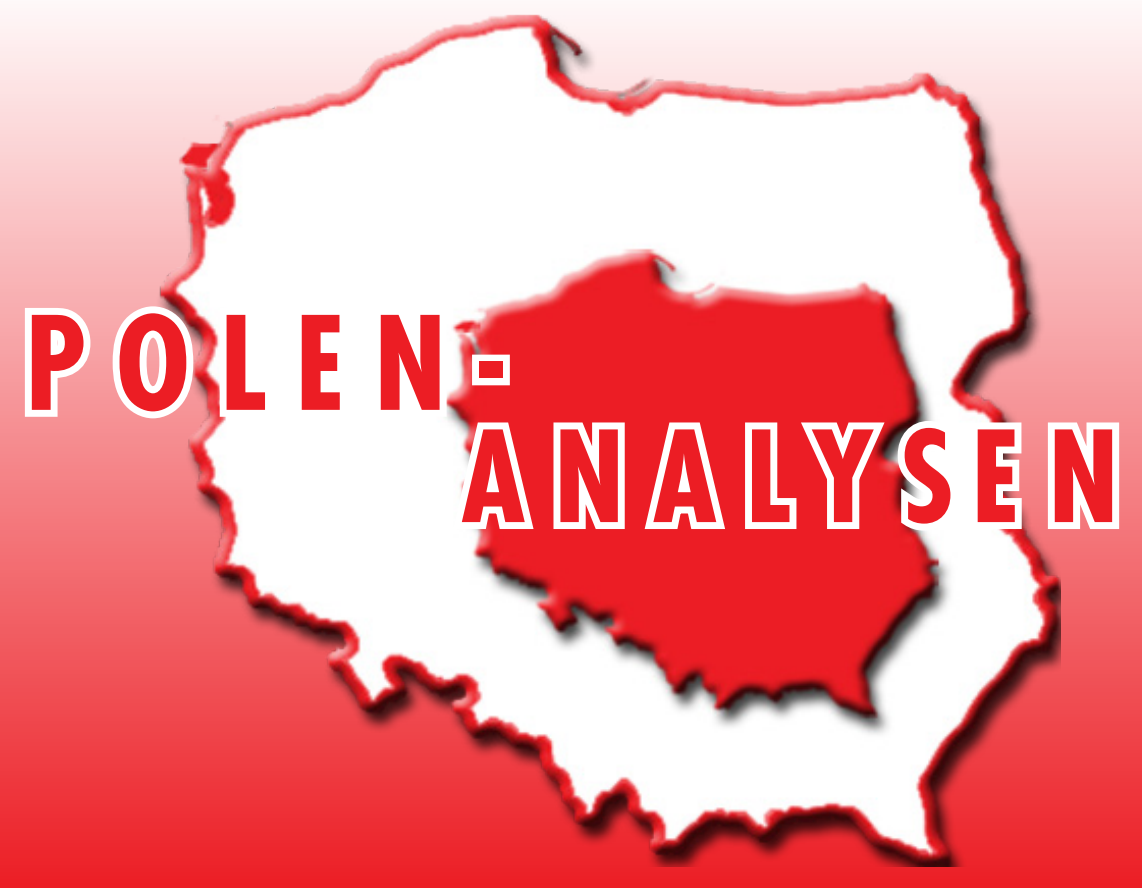

www.laender-analysen.de/polen

\title{
DIE BEWEGUNG FÜR DIE AUTONOMIE SCHLESIENS (RAŚ)
}

ANALYSE

Die Bewegung für die Autonomie Schlesiens (RAŚ)

Marek S. Szczepański, Kattowitz, und Anna Śliz, Oppeln

- TABELLEN UND GRAFIKEN ZUM TEXT

National-ethnische Identifikation in Polen 2011 (Volkszählung)

- KARTEN

Schlesien im 20. Jahrhundert

CHRONIK

Vom 19. Juni bis zum 2. Juli 2012

Die nächste Nummer der Polen-Analysen erscheint nach der Sommerpause am Dienstag, den 18. September 2012.

Die Redaktion wünscht ihren Lesern eine erholsame Sommerzeit. 


\section{Die Bewegung für die Autonomie Schlesiens (RAŚ)}

Marek S. Szczepański, Kattowitz, und Anna Śliz, Oppeln

\section{Zusammenfassung}

Dargestellt werden die Geschichte und die aktuelle Rolle der Bewegung für die Autonomie Schlesiens (Ruch Autonomii Ślaska - RAŚ), die im Januar 1990 gegründet wurde. Mit Rückgriff auf die Geschichte Oberschlesiens wird analysiert, auf welcher Grundlage die Idee der Autonomie fußt und welche Rolle die schlesische Identität im Vergleich zu den Programmen anderer schlesischer Organisationen spielt, die sich zum Teil als Interessenvertreter einer nationalen Minderheit verstehen. Beschrieben werden die Grundzüge der Tätigkeit von $R A S$, ihre Hauptakteure und die ideologischen Leitideen. Die Analyse geht davon aus, dass der Umbruch von 1989 und die darauf folgende Transformationsperiode nicht davon befreit, sich mit der Geschichte Oberschlesiens zu befassen, die den besonderen Charakter der Region bis heute prägt und entsprechende gesellschaftliche und politische Entwicklungen zur Folge hat.

$\mathrm{O}$ erschlesien, der Wirkungsort von $R A S$, ist vor dem Hintergrund anderer Regionen in Polen eine spezifische Region. Es ist dies ein Landstrich mit einer ungewöhnlichen und schwierigen Geschichte, der sich in das Schicksal von mehreren Ländern einschreibt, und spezifischen zwischenmenschlichen Beziehungen und Wertesystemen. Seit der Zeit der Schlesischen Piasten (12. Jh.) stieg der Anteil der deutschen Bevölkerung systematisch, was bei den Polen Unzufriedenheit hervorrief, die sich "wie bei den Deutschen auf der linken Seite der Oder« fühlten (Szaraniec 2007: 7). Die Unterordnung der oberschlesischen Herzogtümer unter die Böhmische Krone verband diese Region mit dem Gebiet der böhmisch-deutschen Kultur, in der bis zum 18. Jahrhundert die tschechische Sprache verbindlich war, wobei ab dem 16. Jahrhundert die deutsche Sprache hinzutrat. In Folge der drei Schlesischen Kriege im 18. Jahrhundert fand sich die oberschlesische Region zum größten Teil in den Grenzen Preußens wieder. In der Region lebten Polen, Deutsche, Mähren (Tschechen), Juden sowie national indifferente Bürger (Szaraniec 2007: 7-8). Der Erste Weltkrieg, das Plebiszit und die drei Schlesischen Aufstände hatten zur Folge, dass sich der größere Teil der oberschlesischen Region dann in den Grenzen Polens befand. Polen wurde nach dem Zweiten Weltkrieg ein Teil des sozialistischen Systems, das von der UdSSR dominiert wurde. Die damaligen Machthaber strebten die Integration des kulturell und national ausdifferenzierten Oberschlesien an. Es wurde ein entsprechendes Schul- und Bildungssystem eingeführt und die Medien wurden entsprechend programmiert. Die Kultivierung der Traditionen der nationalen und ethnischen Minderheiten fand ausschließlich im privaten Bereich statt, in häuslicher Umgebung, oft unter dem Schutz der Kirche, vor allem der katholischen, wenn auch nicht ausschließlich. Das öffentliche Leben wurde von der polnischen Nationalkultur dominiert, und die Kultur der
Minderheiten zu zeigen, war infolge der repressiven Politik nur in einem sehr begrenzten Bereich möglich. Erst als Polen den Weg der Freiheit und Demokratie betrat, entstand eine Atmosphäre des kulturellen Pluralismus, dessen Resultat das heutige Oberschlesien mit seiner nicht nur kulturellen, sondern auch politischen Vielfalt ist (Śliz 2009: 158-159). Erst da hat sich gezeigt, dass die bisher national und ethnisch einheitlichen Regionen Polens von verschiedenen Minderheiten bewohnt werden, zu denen man auch die Oberschlesier zählen kann.

\section{Oberschlesien: eine kulturelle und politische Grenzregion}

Die Geschichte Oberschlesiens bestimmt diese Region als eine kulturelle Grenzregion, d. h. im Verhältnis zu den politisch-administrativen Zentren an der Peripherie gelegen, aber gleichzeitig mit einem deutlichen Bewusstsein der eigenen Andersartigkeit. Trotz der Randlage stand diese Region als Industriegebiet neben dem Ruhrgebiet an zweitwichtigster Stelle zunächst in Preußen und später [ab 1871 - d. Red.] dem vereinigten Deutschland. Die Dampfmaschinen, die hier aufgestellt wurden, gehörten zu den ersten auf dem Kontinent, hier wurde die Nivea Creme entwickelt, die Dusche erfunden, und es wurden früh Straßenbahnen in Betrieb genommen. Hier wurde auch eine verbreitete Alphabetisierung festgestellt, und zwölf Bewohner des historischen Schlesien wurden mit dem Nobelpreis in den Bereichen Physik, Chemie und Ökonomie ausgezeichnet.

Die regionale Ausprägung der Kultur ist dabei, wie bereits erwähnt, ein Ergebnis der langjährigen Durchdringung vieler Kulturen und Traditionen unterschiedlicher Herkunft (Staszczak 1978; Kwaśniewski 1982; Rykiel 1990). Eine charakteristische Eigenschaft dieser Grenzregion ist die Tatsache, dass sie im Laufe der Jahrhunderte von unterschiedlichen kulturellen, politisch-administrativen und ökonomischen Systemen 
beeinflusst wurde. Infolge dessen kennzeichnen die hier lebende Bevölkerung uneindeutige und verschiedene nationale Optionen und ist die nationale Indifferenz keine marginale Erscheinung. Die sich ändernde staatliche Zugehörigkeit Schlesiens, die typisch für eine Randregion ist, ist ein wesentlicher und folgenschwerer historischer Prozess. Unternimmt man einen historischen Schnelldurchgang, dann befand sich Oberschlesien in den letzten zehn Jahrhunderten im großmährischen, böhmischen, polnischen, wieder im böhmischen, im österreichischen, preußischen und nach der Volksabstimmung im polnischen, deutschen und tschechischen politischen Einflussgebiet. Eine wesentliche Feststellung ist, dass Schlesien seit frühester Geschichte in kultureller und zivilisatorischer Hinsicht, und nicht nur in geografischer, ein Teil Europas war und dessen Schicksal teilte, wobei es gleichzeitig seine eigene Besonderheit bewahrte.

Die wechselnde staatliche Zugehörigkeit spielte bei der Desintegration und der Dekonstruktion der schlesischen regionalen Gemeinschaft eine besondere Rolle. Man kann sagen, dass das Plebiszit (20. März 1921) und die drei Schlesischen Aufstände (August 1919, August 1920, Mai bis Juli 1921) die Prozesse der Desintegration gewaltig beschleunigten. Die regionale Gemeinschaft, die mit Oberschlesien und den Kleinregionen stark verbunden war und deren nationale Optionen unterschiedlich, teilweise auch national indifferent oder labil waren, wurde zu eindeutigen Erklärungen ihrer nationalen Zugehörigkeit gezwungen. Die Einteilung infolge des Plebiszits war eine im damaligen Mitteleuropa nicht bekannte Vivisektion, denn im Ergebnis politischer Entscheidungen wurde ein territorial und gesellschaftlich geschlossenes Gebiet zerteilt. Der oberschlesische Stammeszusammenhalt wurde dauerhaft und unumkehrbar verletzt. An dieser Stelle sollen nicht die Dilemmata bewertet werden, die mit einer historischen Gerechtigkeit oder den dramatischen Folgen des Ersten Weltkriegs verbunden sind. Solche Dilemmata kann man ganz historiosophisch auf der Ebene der Interessen von Staaten und ganzer Nationen betrachten. Wir stellen nur die Teilung nach dem Ersten Weltkrieg und der Volksabstimmung heraus sowie deren Auswirkungen, die auf regionaler Ebene wahrnehmbar sind.

Mit Blick auf das politische Schicksal Oberschlesiens scheint die Gründung der Woiwodschaft Schlesien nach dem Ersten Weltkrieg die bedeutendste Entscheidung gewesen zu sein. Dies geschah durch ein Gesetz (15. Juli 1920) des Gesetzgebenden Sejm der Republik Polen. Diese administrativ-politische Einheit wurde als Vereitelung der Autonomiebemühungen und der separatistischen Bestrebungen betrachtet, die vorher in den Aktivitäten von Hans Georg von Praschma, Karl Ulitzka, Hans Lukaschek, Ewald Latacz und auch Wojciech Korfanty
(1907 in den Reichstag gewählt), Adam Napieralski und Jan Kapica zum Ausdruck gekommen waren. Sie alle verfolgten - wenn auch aus sehr unterschiedlichen Gründen - in den ersten Jahrzehnten des 20. Jahrhunderts die Ablösung Oberschlesiens von der schlesischen Provinz und die Verleihung eines autonomen Status, zunächst in den Grenzen Preußens, dann in den Grenzen Polens.

Die Woiwodschaft Schlesien begann erst als selbständige administrative Einheit zu funktionieren, als das polnische Militär in den im Plebiszit Polen zugesprochenen Teil einrückte (zwischen dem 17. Juni und dem 4. Juli 1922). Der Gesetzgebende Sejm verlieh der neu geschaffenen Woiwodschaft Autonomie; symbolträchtige Institutionen waren der Schlesische Sejm und der Schlesische Fiskus, eine Art regionales Schatzministerium. Der Schlesische Sejm erhielt breitgefächerte Kompetenzen in der Innenpolitik und dem Alltag der Woiwodschaft, jedoch nicht in Außenangelegenheiten und Angelegenheiten des Militärs. Unterstrichen werden muss auch, dass der Schlesische Sejm, der zumindest formell mit ausgebauten gesetzgeberischen Kompetenzen ausgestattet war, nach dem Maiputsch (1926) durch den einflussreichen Woiwoden Michał Grażyński beschränkt wurde, den die Zentralregierung und Marschall Józef Piłsudski unterstützten (Klich 2007).

Eine eigene Institution war der Schlesische Fiskus und der mit ihm verbundene Grundsatz der Tangente, d. h. die Einbehaltung von zirka 50 Prozent der schlesischen Einnahmen, während die übrigen 50 Prozent der Republik Polen zugeführt wurden. Das Ende der regionalen Autonomie Schlesiens wurde per Gesetz vom 6. Mai 1945 festgelegt. Viele regionale Forscher zweifeln allerdings die Rechtmäßigkeit dieser politischen Entscheidung an, die von den kommunistischen Machthabern der Nachkriegszeit getroffen wurde. Die Transformation Polens in den 1990er Jahren legte erneut die Träume von einer autonomen Region Schlesien frei.

\section{$R A S:$ Akteure und Ideen}

Die Transformation des Systems der Republik Polen brachte wichtige Veränderungen in der gesellschaftlichen und politischen Landschaft der Region Oberschlesien mit sich. Am 14. November 1989 wurde eine deutsch-polnische Erklärung unterzeichnet, in der die Existenz der deutschen Minderheit bestätigt wurde, die vor allem, aber nicht nur, in Oberschlesien lebt. Im Juni 1991 wurde zwischen der Republik Polen und der Bundesrepublik Deutschland der Vertrag über gute Nachbarschaft und freundschaftliche Zusammenarbeit unterzeichnet. Dieses Dokument regelt auch Angelegenheiten der deutschen Minderheit und ihrer Repräsentanten, die ihre kulturelle Identität pflegen und aktive Teilnehmer 
des politischen Lebens wurden und gewählte Vertreter in den Sejm und in die lokalen Gremien entsenden.

Im Ergebnis der politischen Umgestaltungen fiel der Bewegung für die Autonomie Schlesiens (RAŚ) auf der politischen Bühne Oberschlesiens eine wichtigere Rolle zu. Diese Gesellschaft wurde im Januar 1990 von Menschen aus Oberschlesien gegründet, in der ersten Phase existierte sie unter dem Namen Ruch na rzecz Autonomii Ślaska in Rybnik. Im Jahr 2001 entstand die Region Oppeln von $R A S$ mit Sitz in Oppeln (Opole). $R A S$ in Oppeln umfasst die Kreise von $R A S$ auf dem Gebiet der Woiwodschaft Oppeln. Das Gründungskomitee von $R A S$ bestand aus 16 gebürtigen Schlesiern und das Bergwerk Pniówek (im Dorf Pniówek) gilt als der Ort, wo die Bewegung entstand (Kijonka-Niezabitowska 2008: 507-508). Seit dem 27. Januar 2001 ist die Gesellschaft unter dem Namen Ruch Autonomii Ślaska mit Sitz in Rybnik tätig. Seit einigen Monaten hat die Zentrale ihren Sitz in Kattowitz ${ }^{1}$. Heute gehören zu $R A S$ 7.000 Mitglieder, die in der Mehrheit in der Woiwodschaft Schlesien leben. Das grundlegende Ziel, das in den Statuten festgelegt wurde, ist die Gründung einer autonomen Region in den historischen Grenzen Oberschlesiens. Im Statut von $R A S ́$ werden weitere Ziele ausgeführt: "[...] 1. die Erlangung der Autonomie Schlesiens im Kontext einer ausgereiften Dezentralisierung der Republik Polen; 2. die Weiterentwicklung der regionalen Identität unter den Einwohnern Schlesiens und anderer Regionen der Republik Polen; 3. die Gestaltung und Entwicklung einer aktiven zivilgesellschaftlichen Haltung in der Bevölkerung Schlesiens; 4. die Beteiligung an der Integration aller Einwohner Schlesiens ohne Rücksicht auf die ethnische Option; 5 . Aktivitäten mit dem Ziel des Schutzes der Umwelt und des materiellen und geistigen Erbes der Vorfahren; 6. die Verbreitung der Menschenrechte und der bürgerlichen Freiheiten; 7. Aktivitäten zugunsten der europäischen Integration sowie die Förderung von Kontakten und der Zusammenarbeit zwischen den Gesellschaften; 8. Pflege kultureller und wirtschaftlicher Kontakte zur schlesischen Diaspora sowie emotionaler Bindungen zwischen dieser und der Heimat; 9. die Entwicklung und Gestaltung eines positiven Image von Schlesien; 10. die Förderung der vielfältigen schlesischen Sprache und Rede. $\aleph^{2}$ Um die klar formulierten Ziele umzusetzen, ruft $R A S$ seine Mitglieder u. a. zu großem Engagement im öffentlichen Leben auf, zu Tätigkeiten zugunsten der lokalen Gemeinschaften, zur Unterstützung von Aktivitäten in den Bereichen Kultur, Bildung und Jugendarbeit

1 Eintrag in Krajowy Rejestr Sądowy: http:/krs.cors.gov.pl/Pod miot.aspx?nrkrs=0000021371 (05.06. 2012).

2 Statut Ruchu Autonomii Śląska, rozdział II - Cele, środki i metody działania, \$12: www.autonomia.pl (13.06. 2012). sowie dazu, für die Idee der Autonomie und des Regionalismus einzutreten. In diesem Zusammenhang müssen die von $R A S$ organisierten Oberschlesischen Tage des Erbes, die Aktionen zur Rettung postindustrieller Anlagen und zahlreiche (reale und virtuelle) Bildungsveranstaltungen genannt werden.

\section{Die Vorstellungen von Autonomie}

Ideell beruft sich $R A S$ deutlich auf die Autonomie, die die Woiwodschaft Schlesien in der Zeit der II. Republik besaß und die ihr im "Organischen Statut" vom Gesetzgebenden Sejm zugesprochen worden war. Gleichzeitig sind deutliche Referenzpunkte auch die Situation Spaniens in der Gegenwart, insbesondere Kataloniens. Hervorgehoben werden muss, dass $R A S$ sich von separatistischen Ideen verabschiedet hat und die Ansicht vertritt, dass eine breite Autonomie innerhalb der Republik Polen für Schlesien am günstigsten ist. Deshalb stellt $R A S$ die Notwendigkeit einer strukturellen Reform heraus, bei der die regionalen Unterschiede berücksichtigt und das Identitätsgefühl der regionalen, ethnischen und nationalen Gruppen sowie die Bestrebungen, Formen politischer Autonomie zu manifestieren, respektiert werden sollen. Zu diesem Zweck knüpft $R A S ́$ Kontakte mit ähnlichen Gesellschaften in anderen Regionen Polens, der Bewegung für die Autonomie Masurens (Ruch Autonomii Mazur) oder der Bewegung für die Autonomie Podlachiens (Ruch Autonomii Podlasia). In Hinblick auf die Zukunft des alten Kontinents setzt sich $R A S$ für ein Europa der hundert Fahnen ein, was Europa erlauben würde, zu seinen Wurzeln zurückzukehren. »[...] Das Europa der großen Staaten, das man kennt, ist nicht und war nie das natürliche Europa. Es ist das Ergebnis imperialistischer Rivalität und Eroberungen und sowohl militärischer als auch gesellschaftlich-ökonomischer Aggressionen. Ihr Europa ist ein künstliches Europa, das einer umfassenden Erneuerung bedarf. Das echte, natürliche Europa setzt sich aus vielen kleinen Staaten, vielen nationalen Gemeinschaften, Kreisen und freien Städten zusammen, die über die Teilungen und die Unterschiede hinweg die gemeinsame, seit zwei Jahrtausenden geschmiedete Zivilisation verbindet" (Fouéré 1990: 22).

In den ersten Jahren war die Tätigkeit von $R A S$ nicht sehr ausgeprägt, erst unter der Leitung von Jerzy Gorzelik wurden die Aktivitäten der Gesellschaft intensiver. Ein Beispiel dafür sind die jährlich organisierten "Märsche für Autonomie« für Aktivisten und Sympathisanten von $R A S$ in Kattowitz, aber auch in Oppeln (Kijonka-Niezabitowska 2009: 97). Hervorgehoben werden muss - darauf wies einer der Aktivisten von $R A S$ in Oppeln hin-, dass $R A S ́$ einen ausschließlich regionalen Charakter hat, dessen grundlegendes Ziel die administrative Modernisierung Polens in Richtung einer Repu- 
blik der autonomen Regionen ist, was auch in einem

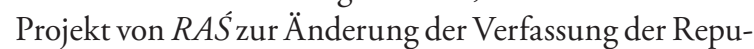
blik Polen zum Ausdruck kam: „Die Verfassung gründet sich auf die unauflösbare Einheit der Republik Polen, auf das gemeinsame und unteilbare Vaterland für alle seine Bürger, zugleich anerkennt und garantiert sie das Recht auf territoriale Autonomie der Regionen, die die Republik Polen bilden, sowie die Solidarität zwischen ihnen allen. Per Gesetz werden auch Einheiten der territorialen Selbstverwaltung gebildet werden, die an der Ausübung der öffentlichen Gewalt [Behörden - d. Red.] teilhaben. ${ }^{3}$ Das Niveau der Autonomie der Regionen wäre den Grundsätzen entsprechend differenziert (spanisches Muster) in: Ausdruck der kulturellen Identität (Sprache, Religion, Tradition) und Grad der wirtschaftlichen Entwicklung. Gleichzeitig sind die Aktivisten von $R A S ́$ bemüht, die Organisation vom nationalen oder ethnischen Stigma zu befreien, wobei alle Gesellschaften mitwirken können, zum Beispiel die Gesellschaft der Menschen Schlesischer Nationalität (Stowarzyszenie Osób Narodowości Ślaskiej-SONŚ), die amtlich registriert ist und über 600 eingetragene Mitglieder hat (gegenwärtig stellt die Staatsanwaltschaft in Oppeln die Rechtmäßigkeit der Registrierung von SONŚ infrage). Ziel von SONŚ ist die Pflege der Tradition, der Sprache und ethnischer und nationaler Elemente derjenigen, die sich mit der schlesischen Nationalität identifizieren: "[...] die Idee, an die polnische Regierung und das polnische Parlament mit einem Antrag auf Anerkennung der ethnischen Minderheit heranzutreten. Das Ergebnis der Volkszählung im vergangenen Jahr, bei der über 800.000 Personen ihre Zugehörigkeit zur schlesischen Nationalität oder zu einer ethnischen Gruppe angaben, sollte in der polnischen Gesetzgebung berücksichtigt werden. « $^{4}$ Die schlesische nationale Identität hatten damals 809.000 Personen angegeben, davon 362.000 ausschließlich die schlesische Identität, und 415.000 sowohl die schlesische als auch die polnische Identität. 418.000 nannten die schlesische Identität an erster Stelle. SONŚ will mit solchen Erklärungen, die die Richtung ihrer Aktivitäten vorgeben, alle Fragen in Sachen Minderheit aus den Aktivitäten von $R A S$ herauslösen und $R A S$ ausschließlich das regionale Engagement überlassen. Inzwischen geht es SONŚ nicht allein um die Anerkennung der Schlesier als ethnische Minderheit, sondern auch darum, dem Schlesischen den Status einer Regionalsprache zu verleihen. Im in Polen geltenden »Gesetz

3 Projekt zmian Konstytucji RP z dnia 2 kwietnia 1997. Rozdział I "Rzeczypospolita« art. 3. pkt. 1 i 2, www.autonomia.pl/n/konsty tucja (13.06. 2012).

4 Ślązacy, łączmy się!, "Jaskółka Śląska. Miesięcznik Górnośląskich Regionalistów«, maj/2012; Ponad 800 tys. Ślązaków, "Gazeta Wyborcza«, 23 marca 2012, S. 4. über nationale und ethnische Minderheiten und über die Regionalsprache" werden die Schlesier nicht als ethnische Minderheit aufgeführt und Schlesisch nicht als Regionalsprache, anders als die kaschubische Sprache [vgl. Polen-Analysen Nr. 95 - d. Red.]. ${ }^{5}$ Im Sejm dauern die Arbeiten an einer Gesetzesnovelle über den Eintrag einer Regionalsprache in Hinblick auf die Anerkennung der schlesischen Sprache als Regionalsprache an. Sie würde die Kriterien einer Regionalsprache, die in der Europäischen Charta der Regional- und Minderheitensprachen (1992) angegeben sind, erfüllen. Darin heißt es, dass eine Regionalsprache traditionell von den Bürgern des betreffenden Staates benutzt wird, die dort eine Minderheit stellen; sie ist kein Dialekt der offiziellen Sprache, also im konkreten Fall des Polnischen, und auch nicht die Sprache von Immigranten.

\section{Die Frage der schlesischen Nationalität}

In diesem Zusammenhang muss der Bund der Bevölkerung Schlesischer Nationalität (Związek Ludności Narodowości Ślaskiej-ZLNŚ) erwähnt werden, dessen gerichtliche Registrierung (Kattowitz, 24. Juni 1997) vom damaligen schlesischen Woiwoden infrage gestellt wurde. Die Registrierung wurde aufgehoben und der Kampf um die erneute Anerkennung von ZLNŚ ging an den Europäischen Gerichtshof für Menschenrechte in Straßburg. Dieser urteilte im Februar 2004, dass von Seiten der polnischen Gerichte keine Rechtsverletzung bestanden hätte, als sie ZLNŚ die Registrierung entzogen haben. Die Entstehung von ZLNŚ und dessen Aktivitäten stießen auf starken Widerspruch vor allem in Schlesien. Ausdruck dessen ist die Entstehung der Bürgerbewegung Polnisches Schlesien (Ruch Obywatelski Polski Ślask-ROPŚ), die am deutlichsten gegen ZLNŚ auftritt (Kijonka-Niezabitowska 2009: 98-99).

Als einschneidende Entwicklungen im Kampf um die Registrierung von ZLNŚ sind die Ergebnisse der Volkszählung von 2002 zu betrachten, als 173.000 Personen zum ersten Mal die Zugehörigkeit zur schlesischen Nationalität angegeben hatten, und aus dem Jahr 2011, als dies 809.000 Menschen taten. In diesem Zusammenhang lassen sich auch in der Haltung der führenden Köpfe von $R A S ́$ Unterschiede in der Akzentuierung des strategischen Vorgehens der Organisation wahrnehmen. So stellt Krzysztof Kluczniok fest. »[...] die Frage der schlesischen Nationalität gehört nicht zu den wichtigsten Zielen von $R A S$. Das Hauptziel ist ein autonomes Oberschlesien in einem Polen der autonomen Regionen. Die Bedingungen sind die Entwicklung der Demokratisierung des Landes, die Dezentralisierung von Ent-

5 Ustawa o mniejszościach narodowych i etnicznych oraz języku regionalnym z dnia 6 stycznia 2005 roku. 
scheidungen und Finanzen, die authentische Selbstverwaltung der Regionen. Daher sind konstitutionelle und administrative Änderungen notwendig, u. a. die Reduzierung der Anzahl der Woiwodschaften, das Ende der zweigliedrigen Struktur von Sejmik [Woiwodschaftsparlament - d. Red.] und Woiwode." (Kijonka, 2005, S. 125) Des Weiteren weist er auf Folgendes hin: "Verwechseln wir nicht $R A S ́$ und ZLNŚ. In $R A S ́$ ist nicht nur für Schlesier Platz. Das wichtigste sind die Verbindung zur Region und das Engagement für das Wohl der Region und ihrer Einwohner. ZLNŚ stützt sich auf das Gefühl der nationalen Besonderheit." (Kijonka 2005, S. 164, vgl. auch Sekuła 2010) Auch die übrigen Aktivisten von $R A S$, u. a. in Oppeln, unterstreichen die deutliche Abgrenzung des Engagements zugunsten des kulturellen Status der schlesischen Minderheit und ihrer Identität von der Idee der autonomen Regionen innerhalb der Republik Polen. Hier kann man von einer deutlichen Trennung von $R A \dot{S}$ und $Z L N S ́$ sowie zwischen $R A S ́$ und $S O N S ́$ sprechen.

\section{Die Gestalt eines autonomen Schlesien}

$R A S$ versammelt Personen, die sich mit den kulturellen und zivilisatorischen Werten der Region verbunden fühlen, die aber nicht notwendig einheimischer Herkunft sind. Im »Informationsbulletin« von RAŚ liest man, dass das Ziel ist, "[...] dass Schlesien alle ihm zustehenden Rechte erhält, die sich vor allem aus dem wirtschaftlichen Potential, den Schätzen der Natur dieser Region, Mineralien und Kohle, herleiten. Der Weg, den wir beschreiten, gründet sich auf das Recht der Menschen dieses Landstrichs, über ihr Schicksal zu entscheiden.» $R A S ́$ vertritt von Beginn an eine klare und deutliche Meinung zur Gestalt eines autonomen Schlesien und zu den Bedingungen seines Bestehens. Die Grundlage für künftige Entscheidungen zur Regionalisierung in diesem Teil Polens sollte nach Meinung von $R A S$ die Wiederherstellung der Autonomie Schlesiens in Anlehnung an das Verfassungsgesetz vom 15. Juli 1920, insbesondere Art. 4 und 5 sein. Ersterer bestimmte die gesetzgeberischen Kompetenzen des Schlesischen Sejm; letzterer die Kompetenzen des Schlesischen Sejm im Bereich Finanzwesen (Schlesischer Fiskus). Ein Anhang zu Art. 5 legte fest, dass ein Teil der schlesischen Einnahmen für gesamtstaatliche Zwecke abgeführt und dass ein entsprechender Teil der Einnahmen in Schlesien dafür zurückgelegt werden musste. $R A S$ bemüht sich außerdem darum, den künftigen Woiwoden von den Einwohnern der Region wählen und nicht durch Benennung durch den Vorsitzenden des Ministerrats der Republik Polen bestimmen zu lassen. Zu den Zielen von $R A S$ hat sich der Vorsitzende Jerzy Gorzelik wiederholt geäußert: "RAŚ strebt ausschließlich nach einer breiten regiona- len Autonomie Ober- und Niederschlesiens im Rahmen von zwei administrativen Einheiten. Der Bezug zu Niederschlesien hat eher symbolische Bedeutung, denn dort gibt es einige Dutzend $R A S$-Mitglieder und an eine politische Massenbewegung ist noch nicht zu denken. Das, was für die Mehrheit der Akteure von $R A S ́$ am wichtigsten ist, ist die Autonomie Oberschlesiens in den historischen Grenzen, das ist vor allem die Schaffung einer Woiwodschaft Oberschlesien in Grenzen, die den historischen Grenzen am nächsten kommen, das heißt, dies hätte mit der aktuellen administrativen Einteilung

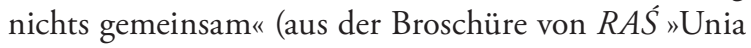
Europejska...»). Damit korrespondieren Aussagen zur Änderung der Grenzen der gegenwärtigen Woiwodschaften Schlesien und Oppeln, die von Vertretern von $R A S$ in den Medien oder in direkten Gesprächen getätigt wurden. In Anlehnung an die Ergebnisse der Volksabstimmungen in den Landkreisen der Woiwodschaft Oppeln geht es um die Aufösung der Woiwodschaft Oppeln und die Integration bestimmter Landkreise entsprechend dem Willen der Einwohner - in die Woiwodschaft Schlesien bzw. Oberschlesien. Es ergibt sich natürlich die Frage, ob die Wiederherstellung der schlesischen Region in den historischen Grenzen noch sinnvoll ist. Rechtfertigen die Veränderungen, die in den letzten Jahrzehnten eintraten, die Bevölkerungsmigration, die Zusammensetzung der Gesellschaft, ein solches Vorhaben? Diese offensichtlichen und grundlegenden Fragen erfordern eine gesellschaftliche Debatte und politischen Willen der regionalen und zentralen politisch Verantwortlichen.

Eine solcherart fundierte autonome Region Oberschlesien hätte die Ergebnisse der Volksbefragungen hinter sich und gleichzeitig die erwünschte/erhoffte Rückkehr zur vergangenen wirtschaftlichen Kraft und Rolle Schlesiens vom Anfang des 20. Jahrhunderts. Die ersten Aktivitäten in Hinblick auf die Entstehung einer autonomen, großen Region Schlesien reichen in das Jahr 1991 zurück, als $R A S S »[\ldots]$ aus begründeter tiefer Sorge um das vorsätzlich ruinierte Schlesische Land und die dauerhafte Verarmung seiner fleißigen Einwohner [...]« einen offenen Brief an die Woiwoden der damaligen Woiwodschaften Kattowitz, Oppeln und Bielsko richtete und die Durchführung eines entsprechenden Referendums forderte. Auf der Grundlage seiner Ergebnisse sollten die Grenzen des autonomen Schlesien bestimmt und die Wahlordnung für den Schlesischen Sejm formuliert werden. Gleichzeitig forderten die Aktivisten von $R A S$, die Zahlungen an die Zentralregierung einzustellen "[...] in Anbetracht der notorischen Vergeudung jedes abgeführten Zloty, der Inventarisierung der unrechtmäßig aus Schlesien ausgeführten Güter mit hohem materiellen und künstlerischen Wert sowie der Anerkennung, dass 
die an Schlesiern verübten Verbrechen keiner Verjährung unterliegen." Dies zeigt, dass die Idee eines autonomen Schlesien in seinen historischen Grenzen in gewissen zeitlichen Abständen mit doppelter Kraft wieder auftritt. Gegenwärtig ist dies wieder der Fall.

$R A S ́$ zeigt auch sichtbare Aktivitäten auf internationaler Ebene. Seit 2003 gehört RAŚ zur European Free Alliance (EFA). Diese Organisation »[...] versammelt Parteien, die sich für die Autonomie europäischer Regionen und Minderheiten einsetzen« (aus der Broschüre von $R A S$ »Unia Europejska...»). Im November 2001 wurde $R A S$ der Beobachterstatus zuerkannt. Im Juni 2003 wurde $R A S ́$ nach der statutarischen Frist gleichberechtigtes Mitglied der EFA. RAS hat auch eine Jugendorganisation, die Oberschlesische Jugend (Młodzież Górnoślaska), die seit 2004 besteht. Sie gehört zur European Free Alliance Youth.

\section{Rabelais zum Schluss}

Als großer Erfolg für $R A S$ erwiesen sich die Selbstverwaltungswahlen, die am 21. November 2010 stattfanden. Im Ergebnis entsandte $R A S$, die 8,49 Prozent der Stimmen der wählenden Einwohner der Woiwodschaft Schlesien erhalten hatte, drei Vertreter in den Sejmik der
Woiwodschaft Schlesien. Gleichzeitig wurde der Vorsitzende von $R A S$, Jerzy Gorzelik, Mitglied der Woiwodschaftsleitung. All dies geschah zum ersten Mal, seitdem Polen auf den Weg der Demokratie und freien Marktwirtschaft zurückgekehrt ist.

Eine Analyse der Veränderungen Oberschlesiens, die vor über zwei Jahrzehnten begannen, zeitigt differenzierte Ergebnisse. Sowohl das wirtschaftliche Leben unterlag radikalen Veränderungen als auch die soziale und kulturelle Struktur der Region. Auch die Hauptakteure der politischen Veränderungen haben gewechselt und $R A S$ strebt mit Konsequenz und mit dem Gefühl, eine Mission zu haben, die Umsetzung der Idee von autonomen Regionen an, darunter Oberschlesien in seinem historischen Rahmen vom Anfang des 20. Jahrhunderts. Die Veränderungen in der Region dauern an und zeigen sowohl neue und vorteilhafte Trends als auch negative und ungünstige. »Glücklich ist der Arzt, den sie am Ende einer Krankheit rufen«, meint François Rabelais. Regionale Krankheiten erfordern, auch wenn sie gut diagnostiziert worden sind, noch viele Anstrengungen, um ihre Auswirkungen einzudämmen. Dies gilt auch für die Aktivitäten von $R A S$.

Übersetzung aus dem Polnischen: Silke Plate

Über die Autoren

Prof. Dr. habil. Marek S. Szczepański, Soziologe, ist Professor am Institut für Soziologie der Schlesischen Universität in Kattowitz (Uniwersytet Śląski w Katowicach). Seine Forschungsinteressen sind u. a. die Soziologie der Region und der lokalen Gesellschaften, soziale Veränderungen und Entwicklungen sowie die Soziologie gesellschaftlicher und ethnischer Konflikte.

Prof. Dr. habil. Anna Śliz, Soziologin, ist Professorin am Institut für Soziologie der Universität Oppeln (Uniwersytet Opolski). Ihre Forschungsfelder sind u. a. die Soziologie gesellschaftlicher und ethnischer Probleme, die Soziologie ethnischer Gruppen, soziale Veränderungen und Konflikte und Multikulturalität in der Gegenwart.

\section{Angeführte Literatur}

- Fouéré, Yann. 1990. W kierunku naturalnej Europy. In: T. Miler (Hg.), Ku Europie 100 flag? Wspótczesne regionalizmy europejskie. Tłum. L. Skupnik. Plan Międzynarodowej Konferencji Autonomistów w Rybniku, Rybnik, S. 22.

- Janeczek, Janusz; Szczepański, Marek S. (Hg.) 2006. Dynamika śląskiej tożsamości. Katowice: Wydawnictwo Uniwersytetu Śląskiego.

- Karta, Nr. 21, 1997 (Rok 1920. Wypędzeni. Obraz systemu).

- Kijonka, Justyna. 2005. Narodowość ślaska. Problemy i kontrowersje. (Praca magisterska napisana pod kierunkiem M. S. Szczepańskiego), Katowice.

- Kijonka-Niezabitowska, Justyna. 2008. Ruch Autonomii Ślaska jako wyraz lokalnego kapitału społecznego. In: M.S. Szczepański, K. Bierwiaczonek, T. Nawrocki (Hg.), Kapitały ludzkie i społeczne a konkurencyjność regionów. Katowice: Wydawnictwo Uniwersytetu Śląskiego, S. 505-516.

- Kijonka-Niezabitowska, Justyna. 2009. Z problemów narodowości i tożsamości śląskiej - dylematy i wybory, "Studia Socjologiczne« 4/2009, S. 85-110.

- Klich, Aleksandra. 2007. Bez mitów. Portrety ze Ślaska. Wydawnictwo WAW.

- Kwaśniewski, Krzysztof. 1982. Zderzenie kultur. Tożsamość a aspekty konfliktów i tolerancji. Warszawa: PWN.

- Nijakowski, Lech M. (Hg.) 2004. Nadciagają Ślązacy. Czy istnieje narodowość śląska? Warszawa: Wydawnictwo Naukowe Scholar. 
- Nijakowski, Lech M. 2006. Domeny symboliczne. Konflikty narodowe i etniczne wymiarze symbolicznym. Warszawa: Wydawnictwo Naukowe Scholar.

- Ponad 800 tys. Ślazaków, "Gazeta Wyborcza«, 23 marca 2012.

- Rykiel, Zbigniew. 1990. Region przygraniczny jako przedmiot badań geograficznych, „Przegląd Geograficzny«, Band 62.

- Sekuła, Elżbieta Anna, 2010. Po co Ślazakom potrzebny jest naród? Warszawa: Wydawnictwo Akademickie i Profesjonalne.

- Staszczak, Zofia. 1978. Pogranicze polsko-niemieckie jako pogranicze etnograficzne. Poznań: Instytut Zachodni.

- Szaraniec, Lech. 2007. Wielokulturowość Górnego Śląska. Katowice: Muzeum Śląskie.

- Szczepański, Marek S. (Hg.). 1994. Górnicy górnoślascy - ludzie zbędni, ludzie luźni? Szkice socjologiczne. KrakówKatowice: AMP.

- Szczepański, Marek S. (Hg). 1993. Dilemmas of Regionalism and the Region of Dilemmas. The Case of Upper Silesia. Katowice: Uniwersytet Śląski.

- Ślązacy, łączmy się!, "Jaskółka Śląska. Miesięcznik Górnośląskich Regionalistów«, 05/2012.

- Śliz, Anna. 2009. Śląs: wielokulturowość czy kulturowe zróżnicowanie?, "Studia Socjologiczne« 4/2009, S. $149-166$.

- Unia Europejska szansą rozwoju regionów. Broszura wydana przez Ruch Autonomii Śląska. Bez daty i miejsca wydania.

- Ustawa konstytucyjna z 15 lipca 1920 roku, zawierająca statut organiczny Województwa Śląskiego (Dz. U. R. P. nr 73, poz. 494) www.law.uj.edu/users/khpp/fontesu/1920.htm (2012.06.05).

- Wanatowicz, Maria. 2004. Od indyferentnej ludności do śląskiej narodowości? : postawy narodowe ludności autochtonicznej Górnego Śląska w latach 1945-2003 w świadomości społecznej. Katowice: Wydawnictwo Uniwersytetu Śląskiego.

\section{National-ethnische Identifikation in Polen 2011 (Volkszählung)}

\section{Tabelle 1: Die national-ethnische Identifikation der in Polen lebenden Bevölkerung} (Volkszählung 2011)

\begin{tabular}{|c|c|c|}
\hline Bevölkerung insgesamt & 38.501 .000 & $100 \%$ \\
\hline \multicolumn{3}{|l|}{ national-ethnische Identifikation } \\
\hline ausschließlich polnisch & 35.251 .000 & $91,56 \%$ \\
\hline polnisch und nicht-polnisch & 834.000 & $2,17 \%$ \\
\hline ausschließlich nicht-polnisch & 554.000 & $1,44 \%$ \\
\hline $\begin{array}{l}\text { darin: } \\
\text { zwei ausschließlich nicht-polnische } \\
\text { national-ethnische Identifikationen }\end{array}$ & 38.000 & $0,10 \%$ \\
\hline nicht ermittelt* & 1.862 .000 & $4,84 \%$ \\
\hline $\begin{array}{l}\text { polnische national-ethnische } \\
\text { Identifikation insgesamt }\end{array}$ & 36.085 .000 & $93,72 \%$ \\
\hline $\begin{array}{l}\text { nicht-polnische national-ethnische } \\
\text { Identifikation insgesamt }\end{array}$ & 1.388 .000 & $3,60 \%$ \\
\hline
\end{tabular}

Die Daten wurden im Rahmen der der Volkszählung (Narodowy Spis Powszechny) im Jahr 2011 erhoben.

* Von den Personen, bei denen keine national-ethnische Zugehörigkeit ermittelt wurde, lebten 1.467.000 zwölf Monate oder länger im Ausland.

Quelle: Główny Urząd Statystyczny: Wyniki Narodowego Spisu Powszechnego Ludności i Mieszkań 2011. Podstawowe informacje o sytuacji demograficzno-społecznej ludności Polski oraz zasobach mieszkaniowych. Warszawa 2012. S. 17. http://www.stat.gov.pl/ gus/5840_12766_PLK_HTML.htm (02.07.2012). 
Tabelle 2: Die Ausdifferenzierung der national-ethnischen Identifikation der in Polen lebenden Bevölkerung (Volkszählung 2011)

\begin{tabular}{|c|c|c|c|c|c|}
\hline $\begin{array}{c}\text { national- } \\
\text { ethnische } \\
\text { Identifikation }\end{array}$ & $\begin{array}{l}\text { erstgenannte } \\
\text { Identifikation }\end{array}$ & $\begin{array}{c}\text { darin: } \\
\text { erstgenannte } \\
=\text { einzige } \\
\text { Identifikation }\end{array}$ & $\begin{array}{l}\text { zweitgenannte } \\
\text { Identifikation }\end{array}$ & $\begin{array}{c}\text { insgesamt } \\
\text { (unabhängig } \\
\text { von der } \\
\text { Klassifizierung } \\
\text { als erst- oder } \\
\text { zweitgenannte } \\
\text { Identifikation)* }\end{array}$ & $\begin{array}{c}\text { darin: } \\
\text { zusammen mit } \\
\text { der polnischen } \\
\text { Identifikation } \\
\text { genannt }\end{array}$ \\
\hline insgesamt & 38.501 .000 & 35.767 .000 & 871.000 & 38.501 .000 & $x$ \\
\hline $\begin{array}{l}\text { polnische } \\
\text { Identifikation }\end{array}$ & 36.007 .000 & 35.251 .000 & 78.000 & 36.085 .000 & $\mathrm{x}$ \\
\hline $\begin{array}{l}\text { nicht-polnische } \\
\text { Identifikation }\end{array}$ & 632.000 & 516.000 & 793.000 & 1.388 .000 & 834.000 \\
\hline \multicolumn{6}{|l|}{ darin: } \\
\hline schlesisch & 418.000 & 362.000 & 391.000 & 809.000 & 415.000 \\
\hline kaschubisch & 17.000 & 16.000 & 211.000 & 228.000 & 212.000 \\
\hline deutsch & 49.000 & 26.000 & 61.000 & 109.000 & 52.000 \\
\hline ukrainisch & 36.000 & 26.000 & 12.000 & 48.000 & 20.000 \\
\hline belorussisch & 37.000 & 31.000 & 10.000 & 47.000 & 15.000 \\
\hline Sinti und Roma & 12.000 & 9.000 & 4.000 & 16.000 & 6.000 \\
\hline russisch & 8.000 & 5.000 & 5.000 & 13.000 & 7.000 \\
\hline amerikanisch & 1.000 & 1.000 & 10.000 & 11.000 & 10.000 \\
\hline lemkisch & 7.000 & 5.000 & 3.000 & 10.000 & 3.000 \\
\hline englisch & 2.000 & 1.000 & 8.000 & 10.000 & 8.000 \\
\hline nicht ermittelt & 1.862 .000 & $\mathrm{x}$ & $\mathrm{x}$ & 1.862 .000 & $\mathrm{x}$ \\
\hline
\end{tabular}

Die Daten wurden im Rahmen der der Volkszählung (Narodowy Spis Powszechny) im Jahr 2011 erhoben.

* Die Spalte enthält die Antworten zu zwei Fragen, die in der Volkszählung gestellt wurden, daher übersteigen die Angaben die Gesamtzahl der Bürger.

Quelle: Główny Urząd Statystyczny: Wyniki Narodowego Spisu Powszechnego Ludności i Mieszkań 2011. Podstawowe informacje o sytuacji demograficzno-społecznej ludności Polski oraz zasobach mieszkaniowych. Warszawa 2012. S. 18. http://www.stat.gov.pl/ gus/5840_12766_PLK_HTML.htm (02.07.2012). 


\section{Schlesien im 20. Jahrhundert}

Karte 1: Provinz Schlesien, Königreich Preußen, administrative Gliederung, 1905

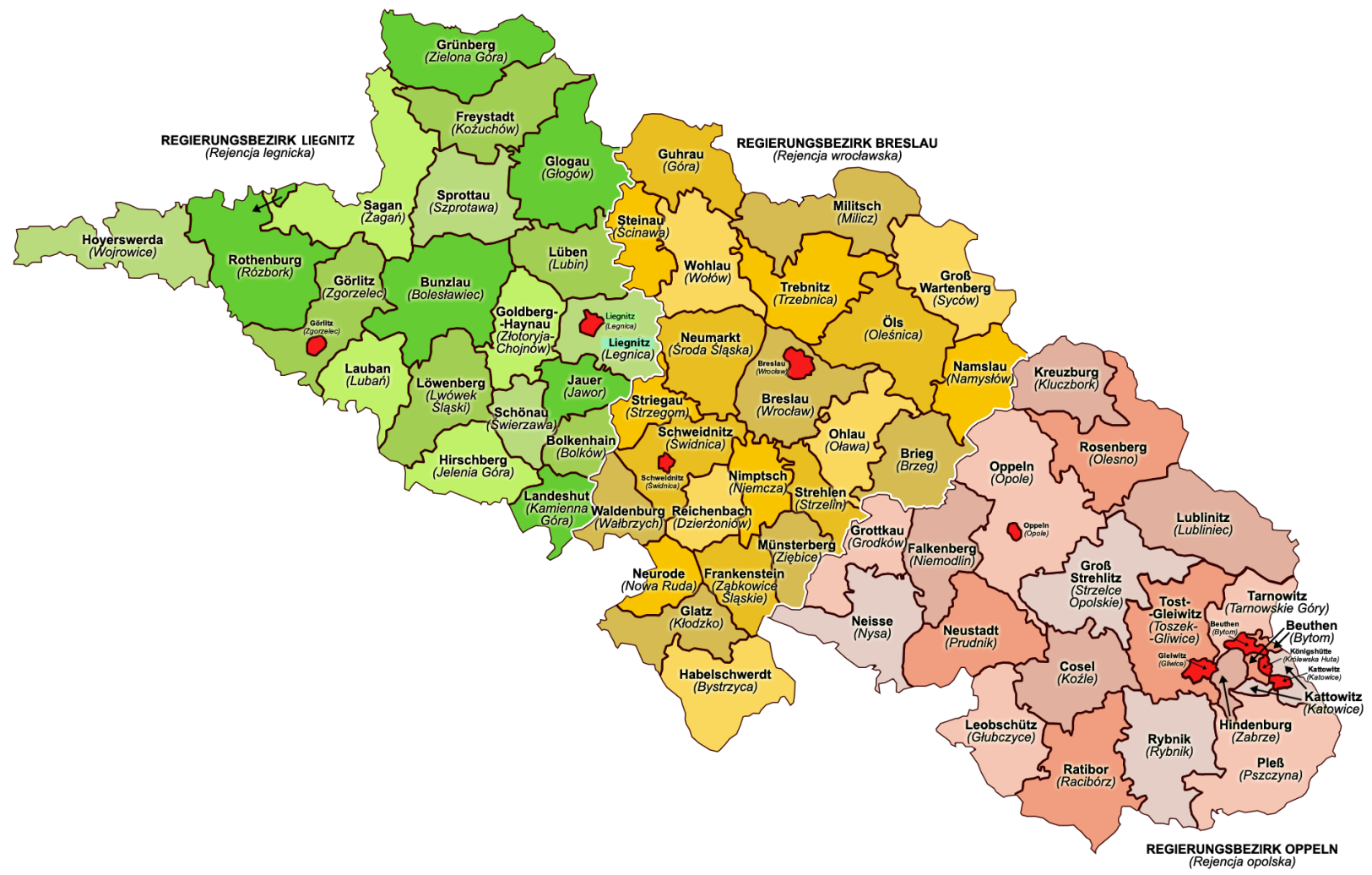

Quelle: http://en.wikipedia.org/wiki/File:Provnice_of_Silesia,_Kingdom_of_Prussia,_1905,_Administrative_Map.png; Karte erstellt von "Qqerim «, mit kleineren Korrekturen durch die Redaktion der Polen-Analysen.

\section{Karte 2: Die Teilung Oberschlesiens 1922}

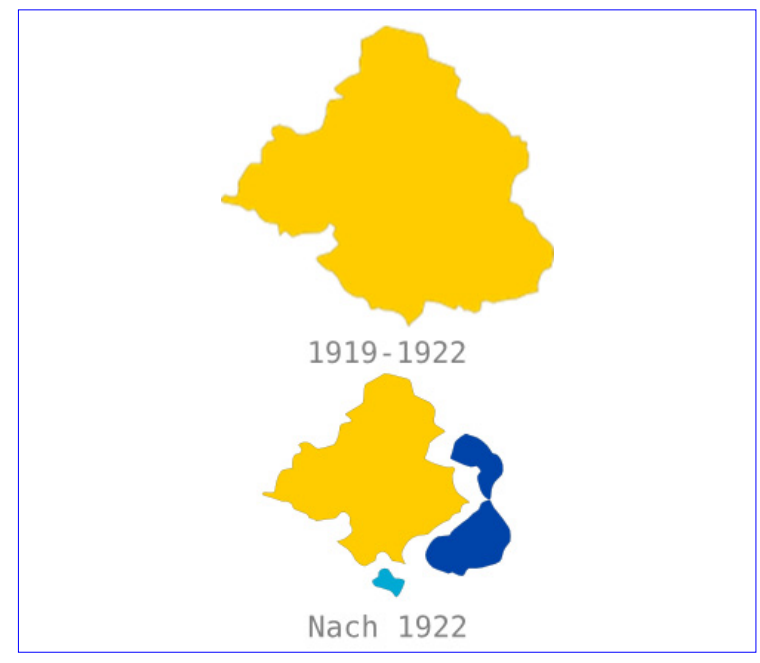

Quelle: http://de.wikipedia.org/wiki/Datei:Teilung_Oberschlesiens.png; Karte erstellt von "Äberlausitzer «; gelb: zu Deutschland, dunkelblau: zu Polen, hellblau: zur Tschechoslowakei.
Karte 3: Die polnischen Woiwodschaften 1922-1939

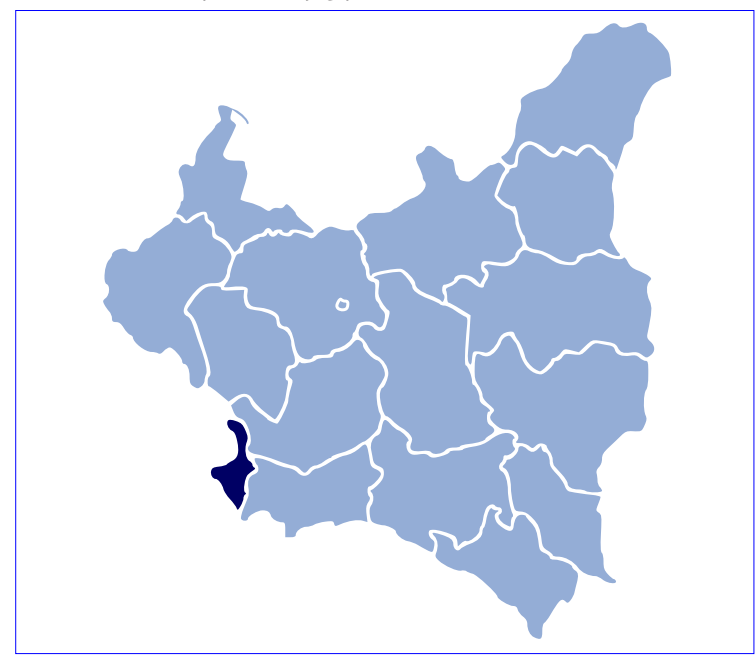

Quelle: http://de.wikipedia.org/w/index.php?title=Datei:Poland_ Voivodeships_adminstrative_division_1930_Silesian_Voivodeship. svg\&filetimestamp=20070124135221; erstellt von "Krzysztof "; dunkelblau: Woiwodschaft Schlesien. 


\section{Karte 4: Die polnischen Woiwodschaften} 1946

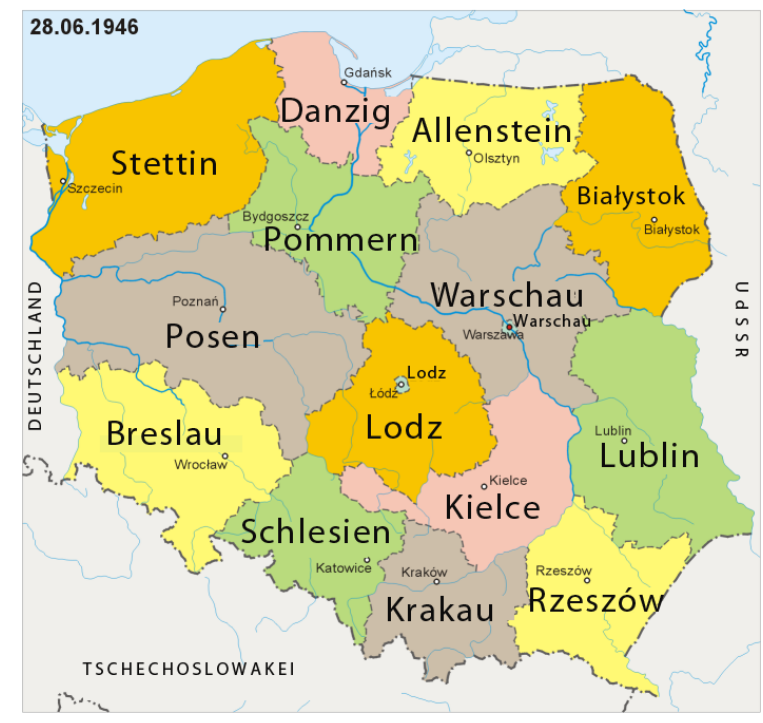

Quelle: http://pl.wikipedia.g-webs.com/w/index.php?title=Plik:POL SKA_28-06-1946.png\&filetimestamp=20090428071830; erstellt von "Aotearoa", Übersetzungen von Ländernamen und Woiwodschaftsbezeichnungen durch die Redaktion der Polen-Analysen.
Karte 5: Die polnischen Woiwodschaften seit 1999

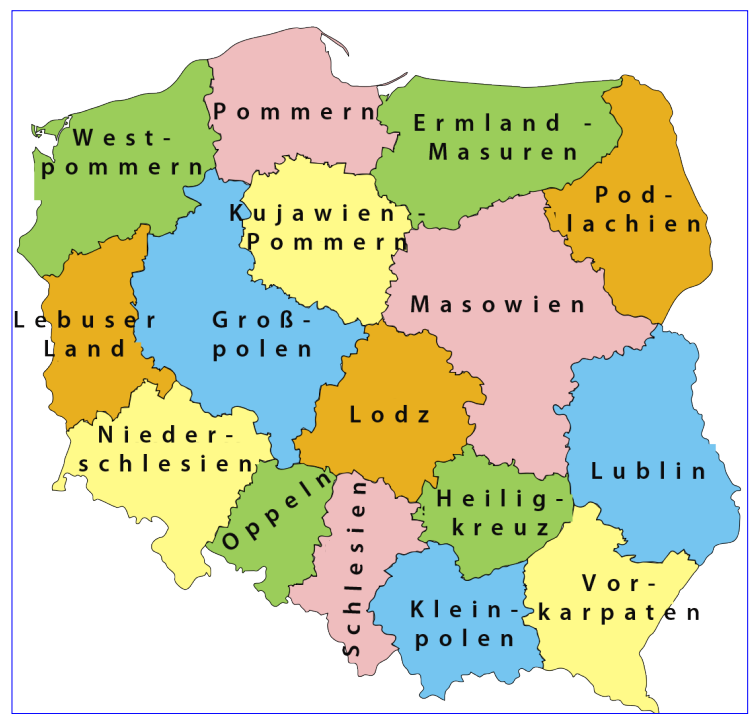

Quelle: http://en.wikipedia.org/wiki/File:Wojewodztwa.svg; erstellt von "odder", Übersetzungen von Woiwodschaftsbezeichnungen durch die Redaktion der Polen-Analysen. 


\section{Vom 19. Juni bis zum 2. Juli 2012}

\begin{tabular}{|c|c|}
\hline 19.06.2012 & $\begin{array}{l}\text { Die Fraktion der Demokratischen Linksallianz (Sojusz Lewicy Demokratycznej - SLD) hat einen Antrag zur } \\
\text { Überprüfung des vom Sejm verabschiedeten neuen Rentengesetzes vorbereitet. Der Antrag wird vom Gesamt- } \\
\text { polnischen Gewerkschaftsverband (Ogólnopolskie Porozumienie Związków Zawodowych - OPZZ) beim Ver- } \\
\text { fassungsgericht eingereicht, da die SLD in Sachen Gesetzesnovelle von Anfang an mit dem OPZZ zusammen- } \\
\text { gearbeitet habe, so der SLD-Vorsitzende Leszek Miller. }\end{array}$ \\
\hline 20.06.2012 & $\begin{array}{l}\text { Nach neuesten Angaben des Statistischen Hauptamts (Główny Urząd Statystyczny - GUS) ist die Industriepro- } \\
\text { duktion im Vergleich zum Vormonat um 4,5\% gestiegen. An der Spitze stehen die Produktion von Chemika- } \\
\text { lien (Anstieg um 15,3\%), elektrischen Geräten (12,5\%) und Lebensmitteln (12,2\%). }\end{array}$ \\
\hline 21.06.2012 & $\begin{array}{l}\text { Der Vorsitzende von Recht und Gerechtigkeit (Prawo i Sprawiedliwość - PiS), Jarosław Kaczyński, teilt mit, } \\
\text { dass er in einem Brief an Ministerpräsident Donald Tusk appelliert habe, sich dafür einzusetzen, dass die Dis- } \\
\text { kriminierung der polnischen Landwirte bei den EU-Direktzahlungen der EU aufhört. }\end{array}$ \\
\hline 22.06.2012 & $\begin{array}{l}\text { Nach der Verhaftung von Andrzej Poczobut in Belarus händigt das polnische Außenministerium dem belarus- } \\
\text { sischen Botschafter in Warschau eine Protestnote aus. Poczobut ist unabhängiger Journalist und Vorsitzender } \\
\text { des Leitenden Rates des Bundes der Polen in Belarus (Związek Polaków na Białorusi), der von der Regierung in } \\
\text { Minsk nicht anerkannt wird. }\end{array}$ \\
\hline 22.06 .2012 & $\begin{array}{l}\text { Der Sejmik (Woiwodschaftsparlament) der Woiwodschaft Schlesien (województwo śląskie) gedenkt mit einer } \\
\text { Feierstunde der Integration eines Teils von Oberschlesien in die wiedererstandene Polnische Republik vor } 90 \\
\text { Jahren. Staatspräsident Bronisław Komorowski mahnt in seiner Rede die polnisch-schlesische Solidarität an und } \\
\text { bezeichnet das Konzept einer autonomen Region als anachronistisch. }\end{array}$ \\
\hline 23.06.2012 & $\begin{array}{l}\text { Grzegorz Schetyna wird zum Vorsitzenden der Bürgerplattform (Platforma Obywatelska-PO) der Region Nie- } \\
\text { derschlesien gewählt. Er hatte die Funktion bereits von } 2006 \text { bis } 2008 \text { inne, bevor er sie wegen seines Amts- } \\
\text { antritts als Innenminister aufgab. Die Wahl wird als Stärkung der Position Schetynas gewertet, der im Okto- } \\
\text { ber } 2009 \text { infolge einer Regierungsumbildung wegen der sogenannten Glücksspielaffäre das Ministeramt abgab. }\end{array}$ \\
\hline 24.06 .2012 & $\begin{array}{l}\text { Der ehemalige Staatspräsident Aleksander Kwaśniewski und der ehemalige Präsident des Europäischen Parla- } \\
\text { ments, Pat Cox, besuchen als Sondergesandte des Europäischen Parlaments die ehemalige Ministerpräsidentin } \\
\text { der Ukraine, Julia Timoschenko, die im vergangenen Jahr wegen Amtsmissbrauch zu sieben Jahren Haft ver- } \\
\text { urteilt worden ist. Zurzeit befindet sie sich im Krankenhaus in Charkiw. Die EU geht von einem politischen } \\
\text { Hintergrund des Urteils aus. }\end{array}$ \\
\hline 25.06 .2012 & $\begin{array}{l}\text { Bei einem mehrtägigen Besuch in Georgien trifft sich Senatsmarschall Bogdan Borusewicz mit dem georgi- } \\
\text { schen Staatspräsidenten Micheil Saakaschwili. Thematisiert werden innenpolitische Angelegenheiten, u. a. die } \\
\text { Parlaments- und Präsidentschaftswahlen im Herbst. Saakaschwili äußert die Bitte, dass Polen Wahlbeobach- } \\
\text { ter entsenden möge, die die Einhaltung der demokratischen Standards kontrollieren sollen. Die Opposition in } \\
\text { Georgien wirft dem amtierenden Staatspräsidenten einen autoritären Regierungsstil vor. }\end{array}$ \\
\hline 26.06.2012 & $\begin{array}{l}\text { Jacek Protasiewicz (Bürgerplattform/Platforma Obywatelska-PO), Vizepräsident des Europäischen Parlaments, } \\
\text { wird in den Vorstand der Europäischen Volkspartei (Christdemokraten) im Europäischen Parlament gewählt. }\end{array}$ \\
\hline 27.06 .2012 & $\begin{array}{l}\text { Die Fraktion der Palikot-Bewegung (Ruch Palikota-RP) beruft eine Parlamentarische Gruppe für einen lai- } \\
\text { zistischen Staat ein. Dies sei die Antwort auf die Unterordnung des Staates unter Kirche, so der RP-Abgeord- } \\
\text { nete Roman Kotliński. }\end{array}$ \\
\hline 28.06.2012 & $\begin{array}{l}\text { Kulturminister Bogdan Zdrojewski teilt nach einem Treffen mit seinem russischen Amtskollegen Wladimir } \\
\text { Medinski in Moskau mit, dass Polen und Russland eine Zusammenarbeit von Archiven zwecks Austauschs von } \\
\text { Archivbeständen aufnehmen werden. Polen hoffe auf die Rückgabe wichtiger Dokumente aus der Zeit der II. } \\
\text { Republik, die während des Zweiten Weltkriegs von der Sowjetunion beschlagnahmt worden waren. }\end{array}$ \\
\hline 29.06.2012 & Nach Angaben des Vorstands der IT-Fachmesse CeBIT wird Polen im Jahr 2013 Partnerland der Messe sein. \\
\hline 01.07.2012 & $\begin{array}{l}\text { Nach einem Treffen mit Vertretern der ukrainischen Opposition in der ukrainischen Hauptstadt Kiew äußert } \\
\text { Staatspräsident Bronisław Komorowski Zweifel, ob die Ukraine in Anbetracht der gegenwärtigen innenpoliti- } \\
\text { schen Probleme und der noch nicht absehbaren Entwicklungen im Zusammenhang mit den Parlamentswahlen } \\
\text { im Herbst weiter die europäische Integration anstrebt. Nach einem Gespräch mit seinem Amtskollegen Viktor } \\
\text { Janukowitsch appelliert er an diesen, konsequent die Integration mit der westlichen Welt zu verfolgen. }\end{array}$ \\
\hline 02.07 .2012 & $\begin{array}{l}\text { Die Pressesprecherin der Kreisstaatsanwaltschaft Warszawa-Praga (Warschau-Praga) teilt mit, dass das zivil- } \\
\text { rechtliche Ermittlungsverfahren zum Flugzeugunglück von Smolensk (2010) eingestellt worden sei. Die orga- } \\
\text { nisatorischen Fehler seien keine strafrechtlichen Vergehen gewesen. }\end{array}$ \\
\hline
\end{tabular}


Die Polen-Analysen erscheinen zweimal monatlich als E-Mail-Dienst. Sie werden gemeinsam vom Deutschen PolenInstitut Darmstadt, der Bremer Forschungsstelle Osteuropa und der Deutschen Gesellschaft für Osteuropakunde herausgegeben.

Ein Archiv der Polen-Analysen finden Sie im Internet unter www.laender-analysen.de/polen

Kostenloses Abonnement unter http://www.deutsches-polen-institut.de/Newsletter/subscribe.php

Diese Analysen finden Sie online als Lizenzausgabe auf

bpb.de

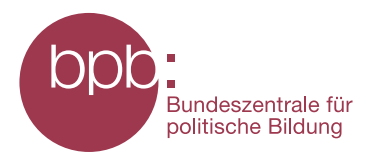

\section{Deutsches Polen-Institut Darmstadt}

Das Deutsche Polen-Institut Darmstadt (DPI) ist ein Forschungs-, Informations-, und Veranstaltungszentrum für polnische Kultur, Geschichte, Politik, Gesellschaft und die deutsch-polnischen Beziehungen, die sich im Kontext der europäischen Integration entwickeln. Das seit März 1980 aktive und bis 1997 von Gründungsdirektor Karl Dedecius geleitete Institut ist eine Gemeinschaftsgründung der Stadt Darmstadt, der Länder Hessen und Rheinland-Pfalz sowie des Bundes. 1987 wurden die Kultusminister der Länder und 2011 das Auswärtige Amt weitere institutionelle Träger. Einen wesentlichen Beitrag zur Verwirklichung der Institutsziele leisten private Stiftungen. Das DPI hat satzungsgemäß die Aufgabe, durch seine Arbeit zur Vertiefung der gegenseitigen Kenntnisse des kulturellen, geistigen und gesellschaftlichen Lebens von Polen und Deutschen beizutragen.

Ziel der Vermittlertätigkeit des DPI ist es, »die zu interessieren, auf die es politisch, wirtschaftlich, gesellschaftlich und kulturell im deutsch-polnischen Verhältnis ankommt« (Leitlinien 1997). Es geht um die Entscheider und Multiplikatoren in Politik, Kultur, Bildung, Verwaltung, Medien und Wirtschaft und, wesentlich stärker ausgeprägt als bisher, um das Hineinwirken in Wissenschaft, Forschung und Bildung.

Derzeit bemüht sich das DPI in Kooperation mit den verstreuten Orten wissenschaftlicher Polen-Kompetenz an deutschen Hochschulen und Forschungsinstituten verstärkt darum, ausgehend von einer Bestandsaufnahme deutscher Polen-Forschung Ort wissenschaftlicher Forschung und verbindendes, vernetzendes und kooperierendes Zentrum zu werden. Ausgangspunkt der Neuausrichtung ist die kaum mehr kontrollierbare Dynamik des Rückbaus der Ressourcen der wissenschaftlichen Polen-Kompetenz in den unterschiedlichen Disziplinen. Mit der über 60.000 Bände zählenden multidisziplinären Fachbibliothek für Polen, die eine einzigartige Sammlung polnischer Literatur in der Originalsprache und in deutscher Übersetzung umfasst, ist das DPI bereits ein geschätzter Ort der Recherche und des wissenschaftlichen Arbeitens. (www.deutsches-polen-institut.de)

\section{Forschungsstelle Osteuropa an der Universität Bremen (www.forschungsstelle.uni-bremen.de)}

1982 gegründet, widmet sich die Forschungsstelle Osteuropa an der Universität Bremen der interdisziplinären Analyse der Länder Ost- und Ostmitteleuropas in Zeitgeschichte und Gegenwart. Der Forschungsschwerpunkt liegt dabei auf der Rolle von "Dissens und Konsens«, von Opposition und Zivilgesellschaft in ihrem historischen, politischen, gesellschaftlichen und kulturellen Kontext. Die Forschungsstelle besitzt in ihrem Archiv eine einzigartige Sammlung alternativer Kulturgüter und unabhängiger Texte aus den ehemaligen sozialistischen Ländern. Darunter befindet sich auch eine umfangreiche Sammlung des "Zweiten Umlaufs», die das Schrifttum und Dokumente unabhängiger Initiativen und gesellschaftlicher Gruppen in Polen aus der Zeit von 1976 bis zum Umbruch umfasst. Hinzu kommt eine umfangreiche Bibliothek mit wissenschaftlicher Literatur. Mit Archiv, Bibliothek und zwei wissenschaftlichen Abteilungen ist die Forschungsstelle auch eine Anlaufstelle sowohl für Gastwissenschaftler als auch für die interessierte Öffentlichkeit.

Eine der Hauptaufgaben der Forschungsstelle ist die Information der interessierten Öffentlichkeit. Dazu gehören unter anderem regelmäßige E-Mail-Informationsdienste für Politik, Wirtschaft, Zivilgesellschaft und Medien. 